\title{
BORDETELLAS, MÁS QUE SOLO PERTUSSIS: GENÉTICA, GENÓMICA, EVOLUCIÓN Y NUEVOS PATÓGENOS PARA LA ESPECIE HUMANA
}

Santiago Sánchez Pardo*, Giovanny Alexander Jácome**, Grégory Alfonso García MD***, Jairo Muñoz Cerón MD, MSc****, Nubia Ponce MSc*****

\section{Resumen}

La Bordetella pertussis es un reconocido patógeno gram negativo relacionado con enfermedad respiratoria aguda en la especie humana. Cada día hay un mayor reporte de cuadros infecciosos producidos por otras bacterias del género Bordetella. Los modelos teóricos actuales y la evidencia científica en relación con la evolución de las distintas bacterias del género, han abierto nuevas posibilidades de comprensión para descifrar los paradigmas en especiación bacteriana, así como los factores genéticos y genómicos involucrados en tal proceso. El objetivo de este artículo es explorar la genética, la genómica y los hallazgos en filogénesis del género Bordetella y difundir el conocimiento actual en relación con el papel nosológico para la salud humana de los nuevos patógenos del grupo.

Palabras clave: Bordetella pertussis, Bordetella sp., evolución, genética, infectología, microbiología, tosferina.

\section{BORDETELLA GENUS, MORE THAN ONLY PERTUSSIS: GENETICS, GENOMICS, EVOLUTION AND NEW HUMAN PATHÓGENS}

\section{Abstract}

Bordetella pertussis is a well known Gram-negative organism that causes acute respiratory disease in humans. There is a trend of an increase in reported cases of infections caused by other bacteria of the Bordetella genus. Current theoretical models and scientific evidence related to the evolution of the various species of this genus have open new possibilities of understanding to solve the paradigms of bacterial speciation, as well as, the genetic and genomic factors involved in such process. The main objective of this article is to explore Bordetella genus genetics, genomics and phylogenetic findings and extend current knowledge on the role of the new strains of this group as human pathogens.

Key words: Bordetella pertussis, Bordetella sp., evolution, genetics, infectology, microbiology, whooping cough.

Fecha recibido: mayo 2 de 2013 - Fecha aceptado: mayo 24 de 2013

* Interno Junior, Facultad de Medicina Unisánitas. Organización Sánitas Internacional. Bogotá DC, Colombia.

** Interno Senior, Facultad de Medicina Unisánitas. Organización Sánitas Internacional. Bogotá DC, Colombia.

*** Experto en Genética, Bioquímica y Biología Celular y Molecular. Experto en Farmacología Terapéutica y Toxicología. Grupo de Medicina Translacional. Facultad de Medicina. Unisánitas. Organización Sánitas Internacional. Bogotá DC, Colombia.

\footnotetext{
***** Experto Microbiología y Enfermedades Tropicales. Facultades de Medicina y Enfermería, Unisánitas. Organización Sánitas Internacional. Bogotá DC, Colombia.

***** Bacterióloga Experta en Microbiología y Laboratorio Clínico. Facultades de Enfermería y Medicina. CMAPS (Centro Multifuncional Avanzado de Prácticas y Simulación). Unsánitas. Organización Sánitas Internacional. Bogotá DC, Colombia.
} 


\section{Introducción}

Pertussis (o tosferina) es un problema de salud pública mundial, dada la dinámica observada durante las últimas décadas. ${ }^{1,2,3}$ Desde el punto de vista epidemiológico tendríamos en promedio 48.5 millones de casos anuales a nivel mundial y 295.000 muertes por año si se proyecta al presente a partir de estadísticas significativas como las del año $2003 .{ }^{4}$

La incidencia de pertussis en Estados Unidos tuvo su máximo pico en 1934, cuando se reportaron 260.000 casos. ${ }^{5}$ Después de la introducción de la vacuna en los años cuarenta del siglo pasado, la más baja incidencia se reportó en 1976 con 1.010 casos. ${ }^{6}$ La vacunación disminuyó la frecuencia casi en un $99 \%$, pero hacia los años ochenta se consolidó un incremento sostenido. ${ }^{7}$ En Colombia, dentro de las bioestadísticas más actuales, para la semana epidemiológica 22 del año 2013, se han notificado 7.209 casos, distribuidos en: 1.751 casos probables, 1.151 casos confirmados por laboratorio, 4.195 casos descartados, 101 casos compatibles y 11 casos confirmados por nexo epidemiológico. Para el 2012 a semana 22 se habían notificado 6.289 casos. $^{8}$

La vacunación ha polarizado un cambio en la incidencia de la patología, tomada inicialmente como un cuadro nosológico de población pediátrica no vacunada, a población vacunada desde el rango de infantes hasta adultos. Este cambio epidemiológico ha sido explicado porque no se siguen los esquemas de vacunación completos y por la inmunidad de corta vida que se ha encontrado con la vacuna acelular. Adicional a ello, una gran variedad de estudios han descubierto, que bajo este nuevo panorama epidémico, los adolescentes y adultos proveen un definitivo reservorio como fuente de mayor transmisión..$^{7,8-12}$

Dentro de los nuevos patógenos que se están encontrando como causa de enfermedad en escenarios muy particulares, son las distintas especies del género Bordetella, muchas de ellas compartiendo similitudes patogénicas. Esto plantea intrigantes preguntas sobre la evolución, la genética y la genómica de estos organismos en conjunto. El objetivo de este artículo de revisión es ofrecer un marco conceptual en relación con estas temáticas y brindar información sobre el rol infectopatogénico de los miembros no pertussis del género Bordetella. ${ }^{13}$

\section{Definiendo el género Bordetella}

Las especies de Bordetella son bacterias del phylum proteobacteria de la subclase beta-proteobacterias (al menos 75 géneros y 220 especies), del orden Burkholderiales, de la familia Alcaligenacea. ${ }^{13-19}$ En la clasificación contemporánea y holística de Cavalier-Smith en la cual se toman en conjunto aspectos fenotípicos y genotípicos, las betaproteobacterias corresponden al linaje filogenético de las Chromatibacterias, compuesto por el complejo unificado de las $\beta$ y $\gamma$ proteobacterias. ${ }^{20-23}$

Los microorganismos de este género se caracterizan por ser cocobacilos gram negativos pleomórficos pequeños entre 0.8 y $0.4 \mu \mathrm{m}$, encapsulados y no productores de esporas. Su metabolismo es aerobio estricto (con la excepción de $B$. petrii que es un anaerobio facultativo). Algunas especies son móviles como B. bronchiseptica, B. avium, B. hinzii y B. trematum. ${ }^{13-19}$

Todas las especies poseen actividad catalasa, oxidan aminoácidos y no fermentan carbohidratos como la glucosa. Su temperatura de crecimiento óptima está en el rango de 35 a $37^{\circ} \mathrm{C}$. Se agrupan por genotipo y se diferencian por fenotipo de acuerdo con pruebas morfológicas, fisiológicas y antigénicas, sobresaliendo el crecimiento en agar McConkey, motilidad, tipos de ácidos grasos, variedad de isoprenoide-quinonas, catalasa, oxidasa (reactivo de Kovács o de Gaby-Hadley), actividad ureasa, producción de pigmento pardo y asimilación de citrato. . $^{1,3,17-19}$

B. pertussis es el miembro del género más sensible y con el más lento crecimiento in vitro, el cual es inhibido por ácidos grasos, iones metálicos, súlfidos y peróxidos. El aislamiento de $B$. pertussis requiere un medio que contenga sustancias denominadas protectivas como el carbón vegetal, sangre o almidón. El medio tradicional de cultivo es el Bordet-Gengou cuya base es almidón de 
papa. Otras especies son menos exigentes y pueden crecer en agar sangre o agar MacConkey. El medio con base de carbón de Regan-Lowe suplementado con glicerol, peptonas y sangre de caballo u oveja, puede también usarse y provee mejor aislación y cultivo que Bordet-Gengou. ${ }^{1-3,17-19}$

Dentro de las técnicas de genotifipicación de las especies del género Bordetella, algunas con un incipiente pero futuro impacto en diagnóstico y seguimiento, se encuentran la hibridación de ADN (ADN-ADN y ADN-ARN) ADN polimórfico amplificado al azar (del inglés RAPD randomly amplified polymorphic DNA method fingerprint), el contenido de guanina-citosina (GC), la tipificación de fagos, la electroforesis de multilocus de enzimas (MLEE multilocus enzyme electrophoresis), la genómica comparativa de secuencias (16S and 23S rADN), el análisis genómico amplio por medio de chip de ADN (GWAS genome wide analysis for microarray based comparative genome hybridization), la presencia o ausencia de elementos de secuencia de inserción (IS Insertion sequence elements), al igual que su distribución en combinación con polimorfismos de ADN generados y el análisis de patrones de restricción de $\mathrm{ARN}$ ribosomal amplificado (ARDRA amplified ribosomal restriction analysis)..$^{1-3,13-19}$

Dentro del género Bordetella se han determinado al presente nueve especies:

- B. pertussishu (hu, de bacteria adaptada a la especie humana).

- B. parapertussi, 2 subespecies: B. parapertussis $_{o v}$ (ov, de bacteria adaptada a ovinos) y $B$. paraper-

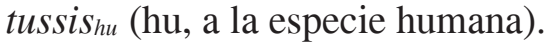

- B. bronchiseptica.

- B. avium.

- B. hinzii.

- B. holmesii.

- B. trematum.

- B. petrii.

- B. ansorpii.

\section{Genética y genómica de la evolución del género Bordetella}

Al presente se ha caracterizado el material genético de siete especies del género Bordetella, en algunos casos como B. pertussis incluyendo varias cepas. ${ }^{24} \mathrm{~A}$ partir de ello se han logrado las siguientes estimaciones:

- El mapeo genético de cinco especies.

- Los genomas de B. pertussis, B. parapertussis y $B$. bronchiseptica, están constituidos por 4.086.186 de pares de bases (bp), 4.773.551 bp y $5.338 .400 \mathrm{bp}$.

- Se han encontrado un total de 3.867-3.873, 4.467 y 5.072 genes para $B$. pertussis, B. parapertussis, y $B$. brochiseptica.

- B. avium posee un genoma de $3.73 \mathrm{Mb}$ (Megabases) y 3.502 genes, y B. petrii 5.29 Mb y 5.089 genes. $^{24-27}$

El análisis genético y genómico comparativo permite determinar que la primera especie del género sobre el planeta fue $B$. petrii y dentro del dendrograma evolutivo un antecesor de $B$. bronchiseptica es la precursora de $B$. pertussis y B. parapertussis. ${ }^{28}$

La evolución dentro del género Bordetella ha sido marcada por la adquisición y la rápida multiplicación, la recombinación cromosómica y la diversificación de los elementos SI (secuencias de inserción) o transposones simples ${ }^{29}$, que corresponden a secuencias génicas móviles, pequeñas (700-2500bp), que no acarrean más información que la requerida para su autorreplicación y movilidad. ${ }^{29}$ Teniendo en cuenta lo anterior, se puede mencionar que:

- $\quad$ B. pertussis tiene 3 SI: IS 481 (238 copias), SI 1.002 (6 copias) e SI 1.663 (17 copias).

- B. parapertussis tiene 2 SI: SI 1.001 (22 copias), IS 1.002 (90 copias).

- B. bronchiseptica: la presencia de elementos varía entre cepas, es así que la cepa RB50 no contienen secuencias SI, pero otras cepas tienen secuencias IS 481, IS 1.001 e IS 1.663. 
La tipificación genética de los distintos tipos SI colaboran en la genotipificación de las especies:

- Varios laboratorios especializados usan la detección de SI 481 para B. pertussis, pero puede también encontrarse en $B$. holmessi.

- La detección de SI 1.001 es indicadora de B. parapertussis, aunque está Presente en cepas de $B$. bronchiseptica y $B$. holmessi.

- El marcador genético SI 1.002 es marcador de cepas de B. pertussis y B. parapertussis. ${ }^{30-34}$

La pérdida de genes específicos en las Bordetellas más antiguas, ha permitido la evolución de nuevas especies filogénicamente hablando. Se puede aseverar esto basándose en el hecho de que $B$. pertussis pierde genes necesarios para la supervivencia como un organismo de vida libre, es por ello que esta especie bacteriana tiene un crecimiento in vitro tan sensible y su incapacidad de crecer en ambientes a temperaturas menores a $37^{\circ} \mathrm{C} . .^{35}$ Dentro de los factores involucrados en la evolución del género también se interroga el papel de la transferencia horizontal a través de bacteriófagos ${ }^{36,37}$, dado el hallazgo de un fragmento de ADN denominado $\mathrm{Bg}$-A que ha sido sólo identificado en $B$. pertussis y es un sistema profago temperado (bacteriófago capaz de existir en forma de profago hacia el interior de una bacteria huésped) que codifica para un putativo sistema de restricción/modificación generador de diversidad..$^{38,39}$ También se interroga si en la evolución del género ha jugado un papel esencial la transmisión y adaptación interespecie diana. ${ }^{40}$

En relación con el contenido guanina-citosina (GC) se ha podido deducir que en los miembros del grupo B.bronchiseptica se halla $66-68 \mathrm{G}+\mathrm{Cmol} \%$, en B. hinzii 65-67G+Cmol\%, en B. trematum $64-65 \mathrm{G}+\mathrm{Cmol} \%$, y $B$. avium posee $62 \mathrm{G}-\mathrm{Cmol} \%$, es entonces claro que $B$. avium es el género relacionado más distante. ${ }^{13,15,23-25,41,42}$ Otra conclusión factible a partir de estos datos es que hay cierta uniformidad genética dentro del género, lo que permite pensar que la especiación dentro de el ha ocurrido hace unos pocos cientos de años. Hay evidencia que sugiere que $B$. bronchiseptica es el ancestro evolutivo más antiguo del grupo, y que las dos especies adaptadas a la especie humana $B$. pertussishu y $B$. parapertussis $_{h u}$ evolucionaron en forma independiente a partir de diferentes linajes. Además B. parapertussis está más relacionado con el grupo $B$. bronchiseptica que $B$. pertussis, permitiendo deducir que $B$. parapertussis es más reciente en su aparición por especiación biológica. ${ }^{13,15,23-25,41-44}$.

La uniformidad observada en el género Bordetella soporta la hipótesis que el fenómeno de especiación puede en verdad ser reciente. Incluso, a favor de ello está el hecho de que la primera observación del cuadro semiológico de Pertussis ocurrió en Francia hacia 1414, la primera epidemia en la historia está fechada hacia 1578 en París, y como tal las características epidémicas y clínicas fueron descritas en 1640 por Guillaume de Baillou. ${ }^{45-48}$

El potencial de colonización y patogenicidad humana de B. pertussis ${ }_{h u}$ y B. parapertussishu, no es en principio mediada por la adquisición de nuevos genes, sino por recombinación del material genético, el cual ha condicionado la generación de pseudogenes (358, 200-220 y 18, respectivamente). Los pseudogenes son remanentes de lo que fueron en algún momento evolutivo previo genes activos, que han sido silenciados por mutaciones, lo cual restringió las condiciones del nicho a colonizar. Muchos pseudogenes se forman a partir de la inserción de elementos SI, lo que se denomina como mutaciones transposónicas. ${ }^{25-27}$

Al comparar los genomas del grupo B. bronchisepti$c a$, el perfil de virulencia y la adaptación a diferentes huéspedes se explica por silenciamiento selectivo de genes. Prueba de esto es que dentro de la genómica comparativa se ha podido concretar la presencia de 358 pseudogenes en el genoma de $B$. pertussis y 200-220 en B. parapertussis, al igual que la pérdida de genes, relacionados con los sistemas de transporte de membrana, metabolismo de pequeñas moléculas y biosíntesis de componentes biomoleculares de superficie. Quizás los dos ejemplos más contundentes son:

- El operón de la toxina Pertussis (PTX) el cual es un islote de patogenicidad bacteriano que contiene 
5 genes (ptxA-ptxE). Si bien se ha ratificado que todas las especies del grupo B. bronchipseptica poseen tal complejo regulador transcripcional, sólo $B$. pertussis lo expresa siempre, y en ocasiones se ha encontrado en las otras dos especies. El análisis genético específico, ha indicado que $B$. bronchiseptica y $B$. parapertussis no tienen un daño estructural del operón en sí, sino del promotor multifuncional de éste (mutaciones inactivantes). Esto sucedió como un proceso de silenciamiento génico mediado por mutaciones o inserción de elementos SI, que originó pseudogenes silentes o deleción total génica. ${ }^{13,15,23,25,35,49,50}$

- Las cepas de B. bronchiseptica son flageladas y móviles. El mismo sistema de genes involucrado con estas funciones se encuentra en el genoma de $B$. pertussis y $B$. parapertussis, el fenómeno entonces es similar al que sucedió con el operón de PTX. ${ }^{13,15,23,25,27,35}$

\section{El grupo B. bronchiseptica: nueva entidad unificada de tres especies y sus diversas cepas especie específicas}

Tomando la información que se ha podido documentar a partir de las técnicas de genotipificación mencionadas, las preguntas sobre la biogénesis evolutiva, el perfil de huésped blanco y el patrón de patogénesis de las distintas especies del género Bordetella generan un sinfín de interrogantes. Dentro de estas tempranas conclusiones hay evidencia concreta de que $B$. pertussis, B. parapertussis y $B$. bronchiseptica comparten características genéticas y genómicas, al igual que ciertas propiedades tales como el hecho de ser patógenos de mamíferos y causar enfermedades de impacto económico importante en la salud humana y en la crianza animal.

Estos tres patógenos tienen en común su adquisición por parte del huésped a través de gotas de secreción ventilorrespiratoria, el fuerte trofismo por los cilios de las células de la mucosa alta, la colonización seguida por proliferación dentro de las células de la mucosa ciliada lo que culmina en ciliostasis, injuria del epitelio, inducción en la liberación de moco y generación de inflamación local. ${ }^{1-3,14-17,19}$ Es por esto, que exíste tendencia actual a comprender a estas tres especies como miembros de un grupo $B$. bronchiseptica. A largo plazo entonces se redefinirá la taxonomía vigente hoy y se tendrá entonces que hablar de $B$. bronchiseptica subspecies pertussis y $B$. bronchiseptica subspecies parapertussis. B. holmessi comparte cierta identidad genética y genómica con $B$. bronchiseptica, B. pertussis y B. parapertussis, pero no las características de virulencia de estas, por lo cual se la excluye del grupo. ${ }^{1-3,13,15,17-19,23,25-27,43,44}$

\section{B. pertussis y B. parapertussis}

B. pertussis o con nomenclatura adecuada en el futuro como B. bronchiseptica subespecie pertussis es un patógeno estricto para la especie humana sin haberse identificado un reservorio animal. $B$. pertussis junto con $B$. parapertussis son los patógenos más comunes del género que se asocian con enfermedad ventilorrespiratoria en la especie humana. B. pertussis es el agente de la tosferina en la especie humana y $B$. parapertussis causa un cuadro similar pero de clínica más moderada. B. parapertussis al igual que $B$. pertussis no tienen un reconocido reservorio ambiental. B. parapertussis es causa de infecciones del tracto ventilorrespiratorio en ovejas, habiéndose identificado una cepa específica denominada como $B$. parapertussis $_{\text {ov. }}{ }^{1-3,16-19,51-53}$

\section{B. bronchiseptica}

B. bronchiseptica es un agente etiológico de enfermedad ventilorrespiratoria en animales. Su papel en infección va desde el extremo de sólo colonización persistente, a una enfermedad debilitante. Una característica peculiar es el hecho de que las cepas especie específicas son más virulentas que aquellas que se comportan como de amplio perfil animal infeccioso animal. Dianas de este agente bacterial son los conejillos de indias, ratas, ratones, zorrillos, zarigüeyas, conejos, mapaches, gatos, perros, hurones, zorros, erizos, ovejas, cerdos, coalas, leopardos, caballos, y gálagos menores (o del Senegal). Ejemplos de zoomorbilidad son la traqueobronquitis en perros denominada 
como tos de las perreras, la rinitis atrófica y bronconeumonía en cerdos, bronconeumonía supurativa con neumonitis intersticial en conejos, y en conejillos de indias se han descrito exudados mucopurulentos o catarrales y afección del oído medio. ${ }^{1-3,16-19,54,55}$ En ocasiones pacientes inmunosuprimidos pueden desarrollar la infección tras la exposición a animales. ${ }^{55-66}$

\section{B. avium}

B. avium es un patógeno en granjas avícolas, causando coriza aviar y rinotraqueitis. Ha sido aislado eventualmente en cultivos de muestras biológicas a partir de pacientes con otitis media crónica. ${ }^{1-3,15-19,67-71}$

\section{B. hinzif}

B. hinzii también es un agente que coloniza el tracto ventilorrespiratorio de animales de granjas avícolas, produciendo la denominada coriza del pavo tipo II. ${ }^{72}$ Existen reportes en la literatura en donde ha sido aislado a partir del esputo de pacientes con fibrosis quística. ${ }^{73,74}$ Se ha asociado como causa de bacteremia en pacientes inmunocomprometidos con síndrome mielodisplásico ${ }^{75}$, en infección por herpesvirus tipo 4 (virus del Ebstein-Barr ${ }^{76}$ ), así como en septicemia en pacientes inmunocompentes ${ }^{77,78}$ y en colangitis crónica en trasplante hepático. ${ }^{79}$

\section{B. holmessi}

B. holmessi ha sido caracterizado por el Centers for Disease Control and Prevention (CDC) de los Estados Unidos, como un miembro del grupo no oxidador 2 (NO2 nonoxidizer group 2). ${ }^{1-3,16-19,80}$ Recibió su nombre en honor al investigador que hizo un variado grupo de aportes a la bacteriología, Barry Holmes. Hay evidencia actual de que su transmisión puede darse de persona a persona, como se pudo ratificar en un reciente estudio japonés. ${ }^{81}$ Se ha encontrado como agente etiológico en cuadros ventilorrespiratorios similares a pertussis. ${ }^{82-87}$

Hay una gran diversidad de reportes de este agente patógeno en distintos escenarios clínicos tales como: bacteremia aislada $^{88}$, bacteremia en asplenia ${ }^{89-91}$, meningitis en asplenia ${ }^{92}$, bacteremia en hemodiálisis ${ }^{93}$, septicemia con endocarditis y falla respiratoria ${ }^{94}$, neumonía adquirida en la comunidad en pacientes con síndrome nefrótico ${ }^{95}$, endocarditis en válvula protésica ${ }^{96}$, pericarditis infecciosa en paciente con linfoma ${ }^{97}$, meningitis en mujer anoréxica ${ }^{98}$, bacteremia en pacientes con anemia de células falciformes ${ }^{99-102}$, artritis séptica en adolescente con anemia hemolítica crónica ${ }^{103}$ y neumonía intersticial y lobar con progresión a fibrosis pulmonar en adolescente inmunocompetente. ${ }^{104}$

\section{B. trematum}

B. trematum ha sido aislada a partir de lesiones ulcerosas cutáneas en diabéticos ${ }^{105}$ y en otitis media. ${ }^{106}$

\section{B. petrii}

Es un patógeno excepcional a la familia por cuanto es un agente anaerobio facultativo. Fue primero aislado de una fuente ambiental (biorreactor anaeróbico enriquecido en sedimento de río ${ }^{107}$ ) y hace poco se reportó como agente causal de mastoiditis supurativa crónica $^{108}$, osteomielitis mandibular ${ }^{109}$ y enfermedad pulmonar crónica (bronquiectasias difusas y persistencia bacteriana mayor a un año). ${ }^{110}$

\section{B. ansorpii}

Primero se aisló en el exudado purulento de un quiste epidérmico. ${ }^{111}$ Después se identificó en pacientes inmunocomprometidos. ${ }^{112}$

\section{Conclusiones}

Los avances en genética y genómica permiten un mayor entendimiento de la dinámica evolutiva de los procariotas (procariontes) y claro ejemplo de ello es la comprensión parcial de la filogénesis del género Bordetella y por extensión de otras familias bacterianas. El conocimiento derivado de ello permitirá una concepción más certera de los fenómenos de variación de los organismos biológicos. Varias de las bacterias del género Bordetella están apareciendo en el panorama 
de salud humana como definitivos agentes patógenos y de ahí la necesidad perentoria del conocimiento de su biología y patobiología por parte del personal de salud. Surgen interrogantes sobre cuál sería el mecanismo de reaparición de esta patología. ¿Obedece a especiación bacteriana, a fallas en las estrategias de inmunoprevención o a ambas?

\section{Referencias}

1. Cherry JD, Heininger U. Pertussis and other Bordetella infections. In: Feigin RD, Cherry J, Demmler-Harrison GJ, Kaplan SL, editors. In: Feigin and Cherry's textbook of pediatric infectious diseases. 6th ed. Philadelphia, PA: W.B. Saunders; 2009. p. 1683-1706

2. Long SS, Edwards KM. Bordetella pertussis (Pertussis) and other species. In: Long SS, Pickering LK, Prober CG. Long's principles and practice of pediatric infectious diseases. 3th ed. Philadelphia, PA: Churchill Livingstone and Elsevier; 2009. p. $858-866$

3. Waters V, Halperin S. Bordetella pertussis. In: Mandell GL, Bennet JE, Dolin R Mandell' Mandell, Douglas, and Bennett's Principles and Practice of Infectious Diseases. 7th ed. Phildelphia, PA(USA):.Churchill Livingstone and Elsevier; 2010. p. 2955-2964.

4. Crowcroft NS, Stein C, Duclos P et al. How best to estimate the global burden of pertussis?. Lancet Infect Dis. 2003 Jul; 3(7):413-8.

5. Centers for Disease Control and Prevention. Pertussis - United States, January 1992-June 1995. MMWR Morb Mortal Wkly Rep. 1995; 44:525-9.

6. Centers for Disease Control and Prevention. Pertussis - United States, 20012003. MMWR Morb Mortal Wkly Rep. 2005; 54:1283-86.

7. Tanaka M, Vitek CR, Pascual FB, et al. Trends in pertussis among infants in the United States, 1980-1999. JAMA. 2003; 290:2968-75.

8. Instituto Nacional de Salud(INS). Boletín Epidemiológico Semanal. Semana epidemiológica número 22 de 2013 (26 de Mayo al 1 de Junio de 2013). [citado 21 jun 2013]. Disponible en: http://www.ins.gov.co/boletin-epidemiologico/Paginas/default.aspx

9. Nelson JD. The changing epidemiology of pertussis in young infants. The role of adults as reservoirs of infection. Am J Dis Child. 1978 Apr; 132(4):371-3.

10. Bisgard KM, Pascual FB, Ehresmann KR, et al. Infant pertussis: who was the source?. Pediatr Infect Dis J. 2004 Nov; 23(11):985-9.

11. Purdy KW, Hay JW, Botteman MF, et al. Evaluation of strategies for use of acellular pertussis vaccine in adolescents and adults: a cost-benefit analysis. Clin Infect Dis. 2004 Jul 1;39(1):20-8

12. Halperin SA. The Control of Pertussis - 2007 and Beyond. N Engl J Med. 2007 Jan 11; 356(2):110-3.

13. Gross R, Keidel K, Schmitt K. Resemblance and divergence: the "new" members of the genus Bordetella. Med Microbiol Immunol. 2010 Aug; 199(3):155-63.

14. Cotter PA, Miller JF. Bordetella. In: Groisman EA, editor. Principles of bacterial pathogenesis. London, UK: Academic Press; 2001. p. 619-74

15. von Wintzingerode F, Gerlach G, Schneider B, Gross R. Phylogenetic relationships and virulence evolution in the genus Bordetella. Curr Top Microbiol Immunol. 2002;264(1):177-99.

16. Mattoo S, Cherry JD. Molecular pathogenesis, epidemiology, and clinical manifestations of respiratory infections due to Bordetella pertussis and other Bordetella subspecies. Clin Microbiol Rev. 2005 Apr; 18(2):326-82.

17. Parton R. Bordetella. In: Borriello SP, Murray PR, Funke G, editors. Topley and Wilson's Microbiology and Microbial Infections. London: Hodder Arnold; 2005. p.1786-1817.

18. Weiss A. The Genus Bordetella. In: Dworkin M, editor. The Prokaryotes: A Handbook on the Biology of Bacteria. $3^{\text {rd }}$ ed. New York: Springer Science, Business Media; 2006. p. 648-74
19. Loeffelholz MJ, Sanden GN. Bordetella pertussis. In: Murray PR, Baron EJ, Jorgensen JH, Landry ML, Pfaller MA, editors. Manual of clinical microbiology. 9th ed. Washington DC: ASM Press; 2007. p. 803-14.

20. Cavalier-Smith T. Only six kingdoms of life. Proc Biol Sci. 2004 Jun 22; 271(1545):1251-62.

21. Cavalier-Smith T. Rooting the tree of life by transition analyses. Biol Direct 2006 Jul. 11; $1: 19$.

22. Cavalier-Smith T. Deep phylogeny, ancestral groups and the four ages of life. Philos Trans R Soc Lond B Biol Sci. 2010 Jan 12; 365(1537):111-32

23. Gerlach G, von Wintzingerode F, Middendorf B, Gross R. Evolutionary trends in the genus Bordetella. Microb Infect. 2001 Jan; 3(1): 61-72.

24. Genome [base de datos en Internet]. Bethesda, MA: NLM, NIH-NCBI; 2009 [citado 15 feb 2013]. Disponible en: http://www.ncbi.nlm.nih.gov/genome/

25. Preston A, Parkhill J, Maskell DJ. The bordetellae: lessons from genomics. Nat Rev Microbiol. 2004 May;2(5):379-90.

26. Parkhill J, Sebaihia M, Preston A, et al. Comparative analysis of the genome sequences of Bordetella pertussis, Bordetella parapertussis and Bordetella bronchiseptica. Nat Genet. 2003 Sep; 35(1):32-40.

27. Sebaihia M, Preston A, Maskell DJ, et al. Comparison of the genome sequence of the poultry pathogen Bordetella avium with those of B. bronchiseptica, B. pertussis, and B. parapertussis reveals extensive diversity in surface structures associated with host interaction. J Bacteriol. 2006 Aug; 188(16):6002-15.

28. Gross R, Guzman CA, Sebaihia M, et al. The missing link: Bordetella petrii is endowed with both the metabolic versatility of environmental bacteria and virulence traits of pathogenic Bordetellae. BMC Genomics. 2008 Sep 30; 9:449.

29. Siguier P, Filée J, Chandler M. Insertion sequences in prokaryotic genomes. Curr Opin Microbiol. 2006 Oct;9(5):526-31.

30. Loeffelholz MJ, Thompson CJ, Long KS, Gilchrist MJ. Detection of Bordetella holmesii using Bordetella pertussis IS481PCR assay. J Clin Microbiol. 2000 Jan; 38(1):467.

31. van der Zee A, Agterberg C, van Agterveld M, Peeters M, Mooi FR. Characterization of IS1001, an insertion sequence element of Bordetella parapertussis. J Bacteriol. 1993 Jan; 175(1):141-7.

32. Templeton KE, Scheltinga SA, van der Zee A, et al. Evaluation of real-time PCR for detection of and discrimination between Bordetella pertussis, Bordetella parapertussis, and Bordetella holmesii for clinical diagnosis. J Clin Microbiol. 2003 Sep;41(9):4121-6.

33. Friedman LE, Messina MT, Santoferrara L, Santillán MA, Mangano A, Franco MA. Characterization of Bordetella bronchiseptica strains using phenotypic and genotypic markers. Vet Microbiol. 2006 Oct 31;117(2-4):313-20.

34. Register KB, Sanden GN. Prevalence and sequence variants of IS481 in Bordetella bronchiseptica: implications for IS481-based detection of Bordetella pertussis. J Clin Microbiol. 2006 Dec; 44(12):4577-83.

35. Cummings CA, Brinig MM, Lepp PW, et al. Bordetella species are distinguished by patterns of substantial gene loss and host adaptation. J Bacteriol 2004; 186:1484-92.

36. Doulatov S, Hodes A, Dai L, Mandhana N, et al. Tropism switching in Bordetella bacteriophage defines a family of diversity-generating retroelements. Nature 2004; 431:476-81.

37. Medhekar B, Miller JF. Diversity-generating retroelements. Curr Opin Microbiol. 2007;10: 388-95.

38. Rauch HC, Pickett MJ. Bordetella bronchiseptica bacteriophage. Can J Microbiol. 1961; 7: 125-33.

39. Middendorf B, Gross R. Representational difference analysis identifies a strainspecific LPS biosynthesis locus in Bordetella spp. Mol Gen Genet. 1999; 262: $189-98$.

40. Shina A, Hart CA, Stenton MD, et al. Distribution of fim 3 and flaA TTGE sequence types amongst isolates of Bordetella bronchiseptica from different host animals. J Med Microbiol. 2002; 51:557-63. 
41. Spears PA, Temple LM, Miyamoto DM, et al. Unexpected similarities between Bordetella avium and other pathogenic Bordetellae. Infect Immun. 2003; 71:2591-7.

42. Register KB, Sacco RE, Nordholm GE. Comparison of ribotyping and restriction enzyme analysis for inter- and intraspecies discrimination of Bordetella avium and Bordetella hinzii. J Clin Microbiol. 2003; 41:1512-9.

43. Bjørnstad ON, Harvill ET. Evolution and emergence of Bordetella in humans. Trends Microbiol. 2005;13: 355-9

44. Diavatopoulos DA, Cummings CA, Schouls LM, et al. Bordetella pertussis, the causative agent of whooping cough, evolved from a distinct, human-associated lineage of B. bronchiseptica. PLoS Pathog. 2005; 1(4):e45.

45. Sydenham T. Opera Universa Medica. London, UK: Sydenham Society; 1741.

46. Bordet J, Gengou O. Le microbe de la coqueluche. Ann Inst Pasteur. 1906; 20:731-41.

47. Lapin JH. Whooping Cough. Springfield, Ill: Charles C. Thomas; 1943.

48. Cone Jr. TC. Whooping cough is first described as a disease sui generis by Baillou in 1640. Pediatrics. 1970; 46: 522.

49. Gross R, Aricò B, Rappuoli R. Genetics of pertussis toxin. Mol Microbiol 1989;3:119-24.

50. Antoine R, Raze D, Locht C. Genomics of Bordetella pertussis toxins. Int J Med Microbiol 2000;290:301-5.

51. Heininger U, Stehr K, Schmitt-Grohé S, et al. Clinical characteristics of illness caused by Bordetella parapertussis compared with illness caused by Bordetella pertussis. Pediatr Infect Dis J. 1994;13:306-9.

52. Porter JF, Connor K, Donachie W. Isolation and characterization of Bordetella parapertussis-like bacteria from ovine lungs. Microbiology. 1994; 140(Pt 2):255-61.

53. Kerr JR, Matthews RC. Bordetella pertussis infection: pathogenesis, diagnosis, management, and the role of protective immunity. Eur J Clin Microbiol Infect Dis. 2000;19:77-88.

54. Switzer WP, Maré CJ, Hubbard ED. Incidence of Bordetella bronchiseptica in wildlife and man in iowa. Am J Vet Res. 1966; 27:1134-6.

55. Goodnow RA. Biology of Bordetella bronchiseptica. Microbiol Rev. 1980; 44:722-38.

56. Borràs Sans M, Bonal J, Bonet J, et al. Bordetella bronchiseptica septicemia in a hemodialysis patient. Nephron. 1991; 59: 676

57. Decker GR, Lavelle JP, Kumar PN, et al. Pneumonia due to Bordetella bronchiseptica in a patient with AIDS. Rev Infect Dis. 1991; 13:1250-1.

58. Woolfrey BF, Moody JA. Human infections associated with Bordetella bronchiseptica. Clin Microbiol Rev. 1991; 4:243-55

59. Ng VL, Boggs JM, York MK, et al. Recovery of Bordetella bronchiseptica from patients with AIDS. Clin Infect Dis. 1992; 15: 376-7.

60. Gueirard P, Weber C, Le Coustumier A, et al. Human Bordetella bronchiseptica infection related to contact with infected animals: persistence of bacteria in host. J Clin Microbiol. 1995; 33:2002-6.

61. Tamion F, Girault C, Chevron V, et al. Bordetella bronchoseptica pneumonia with shock in an immunocompetent patient. Scand J Infect Dis. 1996; 28:197-8.

62. Stefanelli P, Mastrantonio P, Hausman SZ, et al. Molecular characterization of two Bordetella bronchiseptica strains isolated from children with coughs. J Clin Microbiol. 1997;35: 1550-5.

63. Choy KW, Wulffraat NM, Wolfs TF, et al. Bordetella bronchiseptica respiratory infection in a child after bone marrow transplantation. Pediatr Infect Dis J. 1999;18:481-3.

64. Ner Z, Ross LA, Horn MV, et al. Bordetella bronchiseptica infection in pediatric lung transplant recipients. Pediatr Transplant. 2003; 7:413-17.

65. Viejo G, de la Iglesia P, Otero L, et al. Bordetella bronchiseptica pleural infection in a patient with AIDS. Scand J Infect Dis. 2002; 34:628-29.

66. Berkowitz DM, Bechara RI, Wolfenden LL. An unusual cause of cough and dyspnea in an immunocompromised patient. Chest. 2007; 131:1599-1602.
67. Kersters K, Hinz KH, Hertle A, et al. Bordetella avium sp. nov., isolated from the respiratory tracts of turkeys and other birds. Int J Syst Bacteriol. 1984; 34: 56-70.

68. Dorittke C, Vandamme P, Hinz KH, et al. Isolation of a Bordetella avium-like organism from a human specimen. Eur J Clin Microbiol Infect Dis. 1995; 14:451454.

69. Spears PA, Temple LM, Miyamoto DM, et al. Unexpected similarities between Bordetella avium and other pathogenic Bordetellae. Infect Immun. 2003; 71:2591-7.

70. Sebaihia M, Preston A, Maskell DJ, et al. Comparison of the genome sequence of the poultry pathogen Bordetella avium with those of B. bronchiseptica, B. pertussis, and B. parapertussis reveals extensive diversity in surface structures associated with host interaction. J Bacteriol. 2006;188:6002-15.

71. Harrington AT, Castellanos JA, Ziedalski TM, et al. Isolation of Bordetella avium and novel Bordetella strain from patients with respiratory disease. Emerg Infect Dis. 2009; 15:72-4.

72. Register KB, Kunkle RA. Strain-specific virulence of Bordetella hinzii in poultry. Avian Dis. 2009; 53:50-4.

73. Spilker T, Liwienski AA, LiPuma JJ. Identification of Bordetella spp. in respiratory specimens from individuals with cystic fibrosis. Clin Microbiol Infect. 2008; 14: 504-6.

74. Hayashimoto N, Yasuda M, Goto K, et al. Study of a Bordetella hinzii isolate from a laboratory mouse. Comp Med. 2008; 58:440-6.

75. Fry NK, Duncan J, Edwards MT, et al. A UK clinical isolate of Bordetella hinzii from a patient with myelodysplastic syndrome. J Med Microbiol. 2007; 56:1700-3.

76. Hristov AC, Auwaerter PG, Romagnoli M, et al. Bordetella hinzii septicemia in association with Epstein-Barr virus viremia and an Epstein-Barr virus-associated diffuse large B-cell lymphoma. Diagn Microbiol Infect Dis. 2008;61: 484-6.

77. Cookson BT, Vandamme P, Carlson LC, et al. Bacteremia caused by a nove Bordetella species, “B. hinzii.". J Clin Microbiol. 1994; 32:2569-71.

78. Kattar MM, Chavez JF, Limaye AP, et al. Application of $16 \mathrm{~S}$ rRNA gene sequencing to identify Bordetella hinzii as the causative agent of fatal septicemia. J Clin Microbiol. 2000; 38:789-94.

79. Arvand M, Feldhues R, Mieth M, et al. Chronic cholangitis caused by Bordetella hinzii in a liver transplant recipient. J Clin Microbiol. 2004; 42:2335-37.

80. Weyant RS, Hollis DG, Weaver RE et al. Bordetella holmesii sp. nov., a new gramnegative species associated with septicemia. J Clin Microbiol. 1995; 33:1-7.

81. Kamiya H, Otsuka N, Ando Y, et al. Transmission of Bordetella holmesii during Pertussis Outbreak, Japan. Emerg Infect Dis. 2012; 18:1166-9.

82. Yih WK, Silva EA, Ida J, et al. Bordetella holmesii-like organisms isolated from Massachusetts patients with pertussis-like symptoms. Emerg Infect Dis. 1999; 5: 441-3.

83. Mazengia E,Silva EA, Peppe JA, et al. Recovery of Bordetella holmesii from patients with pertussis-like symptoms: Use of pulsed-field gel electrophoresis to characterize circulating strains. J Clin Microbiol. 2000; 38: 2330-3.

84. Guthrie JL, Robertson AV, Tang P et al. Novel duplex real-time PCR assay detects Bordetella holmesii in specimens from patients with Pertussis-like symptoms in Ontario, Canada. J Clin Microbiol. 2010; 48:1435-7.

85. Mooi FR, Bruisten S, Linde I et al. Characterization of Bordetella holmesii isolates from patients with pertussis-like illness in The Netherlands. FEMS Immunol Med Microbiol. 2012; 64:289-91.

86. Njamkepo E, Bonacorsi S, Debruyne M, et al. Significant finding of Bordetella holmesii DNA in nasopharyngeal samples from French patients with suspected pertussis. J Clin Microbiol. 2011;49: 4347-8.

87. Miranda C, Porte L, García P. Bordetella holmesii in nasopharyngeal samples from Chilean patients with suspected Bordetella pertussis infection. J Clin Microbiol. 2012; 50:1505

88. Morris JT, Myers M. Bacteremia due to Bordetella holmesii. Clin Infect Dis. $1998 ; 27: 912-3$

89. Lindquist SW, Weber DJ, Mangum ME, et al. Bordetella holmessi sepsis in an asplenic adolescent. Pediatr Infect Dis J. 1995;14:813-5. 
90. Shepard CW, Daneshvar MI, Kaiser RM, et al. Bordetella holmesii bacteremia: A newly recognized clinical entity among asplenic patients. Clin Infect Dis. 2004; 38:799-804.

91. Panagopoulos MI, Saint Jean M, Brun D, et al. Bordetella holmesii bacteremia in asplenic children: report of four cases initially misidentified as Acinetobacter lwoffii. J Clin Microbiol. 2010; 48:3 762-4.

92. Livovsky DM Md, Leibowitz D, Hidalgo-Grass C, et al. Bordetella holmesii meningitis in an asplenic patient with systemic lupus erythematosus. J Med Microbiol. 2012 Aug; 61(Pt 8):1165-7.

93. Greig JR, Gunda SS, Kwan JTC. Bordetella holmesii bacteraemia in an individual on haemodialysis. Scand J Infect Dis. 2001; 33:716-7.

94. Tang YW, Hopkins MK, Kolbert CP, et al. Bordetella holmesii-like organisms associated with septicemia, endocarditis, and respiratory failure. Clin Infect Dis. 1998; 26:389-92.

95. Dorbecker C, Licht C, Korber F, et al. Community-acquired pneumonia due to Bordetella holmesii in a patient with frequently relapsing nephrotic syndrome. J Infect. 2007; 54:e203-5.

96. Jonckheere S, De Baere T, Schroeyers P, et al. Prosthetic valve endocarditis caused by Bordetella holmesii, an Acinetobacter lookalike. J Med Microbiol. 2012; 61(Pt 6):874-7.

97. Nei T, Hyodo H, Sonobe K, et al. First report of infectious pericarditis due to Bordetella holmesii in an adult patient with malignant lymphoma. J Clin Microbiol. 2012; 50:1815-7.

98. Van Balen T, Nieman AE, Hermans MH, et al. Bordetella holmesii meningitis in a 12-year-old anorectic girl. Pediatr Infect Dis J. 2012; 31:421-2.

99. Njamkepo E, Delisle F, Hagege I, et al. Bordetella holmesii isolated from a patient with sickle cell anemia: analysis and comparison with other Bordetella holmesii isolates. Clin Microbiol Infect. 2000; 6:131-6.

100. McCavit TL, Grube S, Revell P, et al. Bordetella holmesii bacteremia in sickle cell disease. Pediatr Blood Cancer. 2008; 51:814-6.
101. Monnier S, Therby A, Couzon B, et al. Bordetella holmesii bacteremia in a 26-year-old patient with sickle cell disease. Med Mal Infect. 2010; 40:299-301.

102. Barrado L, Barrios M, Sanz F, et al. Bordetella holmesii bacteremia in a child with sickle cell disease. Enferm Infecc Microbiol Clin. 2011; 29:779-80.

103. Moissenet D, Leverger G, Mérens A, et al. Septic arthritis caused by Bordetella holmesii in an adolescent with chronic haemolytic anaemia. J Med Microbiol. 2011; 60(Pt 11):1705-7.

104. Russell FM, Davis JM, Whipp MJ, et al. Severe Bordetella holmesi iinfection in a previously healthy adolescent confirmed by gene sequence analysis. Clin Infect Dis. 2001; 33:129-130.

105. Daxboeck F, Goerzer E, Apfalter P, et al. Isolation of Bordetella trematum from a diabetic leg ulcer. Diabet Med. 2004; 21:1247-8.

106. Vandamme P, Heyndrickx M, Vancanneyt M, et al. Bordetella trematum sp. nov., isolated from wounds and ear infections in humans, and reassessment of Alcaligenes denitrificans Ruger and Tan 1983. Int J Syst Bacteriol. 1996; 46: 849-58.

107. von Wintzingero de F, Schattke A, Siddiqui RA, et al. Bordetella petrii sp. nov., isolated from an anaerobic bioreactor, and emended description of the genus Bordetella. Int J Syst Evol Microbiol. 2001; 51:1257-65.

108. Stark D, Riley LA, Harkness J, et al. Bordetella petrii from a clinical sample in Australia: Isolation and molecular identification. J Med Microbiol. 2007; 56:4357.

109. Fry NK, Duncan J, Malnick H et al. Bordetella petrii clinical isolate. Emerg Infect Dis. 2005; 11:1131-3.

110. Le Coustumier A, Njamkepo E, Cattoir V, et al. Bordetella petrii infection with long-lasting persistence in human. Emerg Infect Dis. 2011;17:612-8.

111. Ko KS, Peck KR, Oh WS, et al. New species of Bordetella, Bordetella ansorpii sp. nov., isolated from the purulent exudate of an epidermal cyst. J Clin Microbiol. 2005; 43: 2516-9.

112. Fry NK, Duncan J, Malnick H, et al. The first UK isolate of 'Bordetella ansorpii' from an immunocompromised patient. J Med Microbiol. 2007; 56: 993-5. 